João Manuel R. S. Tavares

Xiongbiao Luo $\cdot$ Shuo Li

Editors

\title{
Bio-Imaging and
}

Visualization for Patient-

Customized Simulations 


\title{
Contents
}

\author{
A Novel Colon Wall Flattening Model for Computed \\ Tomographic Colonography: Method and Validation . . . . . . . . 1 \\ Huafeng Wang, Lihong Li, Hao Han, Yunhong Wang, Weifeng Lv, \\ Xianfeng $\mathrm{Gu}$ and Zhengrong Liang \\ Biomechanical Simulation of Lung Deformation \\ from One CT Scan . . . . . . . . . . . . . . . . . . . . . 15
}

Feng Li and Fatih Porikli

2D-3D Registration: A Step Towards Image-Guided Ankle Fusion . . .

Ahmed Shalaby, Aly Farag, Eslam Mostafa and Todd Hockenbury

\section{A Graph-Based Methodology for Volumetric Left}

Ventricle Segmentation

S. P. Dakua, J. Abi Nahed and A. Al-Ansari

Minimally Interactive MRI Segmentation for Subject-Specific

Modelling of the Tongue $\ldots \ldots \ldots \ldots \ldots \ldots \ldots$

Negar M. Harandi, Rafeef Abugharbieh and Sidney Fels

Real-time and Accurate Endoscope Electromagnetic Tracking via Marker-free Registration Based on Endoscope Tip Center

Xiongbiao Luo and Kensaku Mori

Evaluation of Image Guided Robot Assisted Surgical Training

for Patient Specific Laparoscopic Surgery . . . . . . . . . . . . .

Tao Yang, Kyaw Kyar Toe, Chin Boon Chng, Weimin Huang,

Chee Kong Chui, Jiang Liu and Stephan K. Y. Chang

Proxemics Measurement During Social Anxiety Disorder

Therapy Using a RGBD Sensors Network

Julien Leroy, François Rocca and Bernard Gosselin 
How Do Sex, Age, and Osteoarthritis Affect Cartilage Thickness at the Thumb Carpometacarpal Joint? Insights from Subject-Specific Cartilage Modeling. . . . . . . . . . . . . . . . 103 Eni Halilaj, David H. Laidlaw, Douglas C. Moore and Joseph J. Crisco

Patient Specific Modeling of Pectus Excavatum for the Nuss Procedure Simulation . . . . . . . . . . . . . . . . . . . . . 113 Krzysztof J. Rechowicz, Mohammad F. Obeid and Frederic D. McKenzie

Formulating a Pedicle Screw Fastening Strength Surrogate via Patient-Specific Virtual Templating and Planning. Cristian A. Linte, Jon J. Camp, Kurt Augustine, Paul M. Huddleston, Anthony A. Stans, David R. Holmes III and Richard A. Robb 